\title{
REAL ANALYTIC SUBMANIFOLDS UNDER UNIMODULAR TRANSFORMATIONS
}

\author{
XIANGHONG GONG
}

(Communicated by Eric Bedford)

\begin{abstract}
We study the normal form of totally real and real analytic submanifolds in $\mathbb{C}^{n}$ under holomorphic unimodular transformations. We also consider the unimodular normal form of real surfaces in $\mathbb{C}^{2}$ near an elliptic complex tangent with the non-vanishing Bishop invariant or near a non-exceptional hyperbolic complex tangent.
\end{abstract}

\section{INTRODUCTION}

In this paper, we study the normal forms of $n$-dimensional real analytic submanifolds in $\mathbb{C}^{n}(n \geq 2)$ under unimodular transformations. By a unimodular transformation we mean a local biholomorphic mapping which preserves the $n$-form $\Omega=d z_{1} \wedge \cdots \wedge d z_{n}$ on $\mathbb{C}^{n}$. For a totally real and real analytic submanifold $M^{k} \subset \mathbb{C}^{n}$ of dimension $k$, we will prove that it is locally equivalent to the standard $\mathbb{R}^{k} \subset \mathbb{C}^{n}$ under unimodular transformations if $k<n$. When $k=n$, there exists an invariant function attached to $M=M^{n}$. To describe this, we fix $p \in M$ and put

$$
\omega_{M}=\left.\Omega\right|_{M}=e^{i \theta} V, \quad \theta(p) \in[0, \pi),
$$

where $\theta$ is a real analytic function and $V$ is a non-vanishing real $n$-form on $M$. We will see that $M$ is locally equivalent to the standard $\mathbb{R}^{k} \subset \mathbb{C}^{n}$ under unimodular transformations defined near $p$ if and only if $\theta$ vanishes. In section 2, we will use Vey's Morse lemma [6] to prove the following:

Theorem 1.1. Suppose that $M \subset \mathbb{C}^{n}(n \geq 2)$ is a totally real and real analytic $n$-dimensional submanifold containing $p$. Let $\theta$ be given by (1.1). If $p$ is a non-degenerate critical point of $\theta$, then there is a unimodular transformaton $\phi$ defined near $p$ with $\phi(p)=0$ such that

$$
\tilde{M}=\phi(M): \operatorname{Im} z_{1}=\operatorname{Im} z_{n-1}=\operatorname{Im}\left\{z_{n} a(z)\right\}=0,
$$

Received by the editors April 19, 1993.

1991 Mathematics Subject Classification. Primary 32F25.

Key words and phrases. Normal form, unimodular transformations, totally real, complex tangent. 
in which $a(z)$ is given by

$$
\begin{gathered}
\frac{\partial}{\partial z_{n}}\left\{z_{n} a(z)\right\}=\exp \left\{-i \sum_{k=0}^{\infty} c_{k} h\left(z_{1}, \ldots, z_{n-1}, z_{n} a(z)\right)^{k}\right\}, \\
h(z)=\sum_{j=1}^{s} z_{j}^{2}-\sum_{j=s+1}^{n} z_{j}^{2}, \quad 1 \leq s \leq n .
\end{gathered}
$$

Moreover, $s$ and coefficients $c_{j}(0 \leq j<\infty)$ give the full set of unimodular invariants of $M$.

We now consider a real analytic surface in $\mathbb{C}^{2}$ with a complex tangent at 0 , which is given by

$$
M: z_{2}=z_{1} \bar{z}_{1}+\gamma z_{1}^{2}+\bar{\gamma} \bar{z}_{1}^{2}+H\left(z_{1}, \bar{z}_{1}\right), \quad \gamma \in \mathbb{C},
$$

in which the power series $H\left(z_{1}, \bar{z}_{1}\right)$ begins with the third-order terms, $\gamma$ is a unimodular invariant, and $|\gamma|$ is the Bishop invariant [2]. The complex tangent of $M$ at 0 is said to be elliptic, parabolic, or hyperbolic according to $0 \leq$ $|\gamma|<1 / 2,|\gamma|=1 / 2$, or $1 / 2<|\gamma|<\infty$, respectively. Let $\lambda$ be one of the roots of $|\gamma| \lambda^{2}+\lambda+|\gamma|=0$. $\quad \gamma$ is said to be non-exceptional if $\lambda$ is not a root of unity. A formal unimodular transformation of $\mathbb{C}^{n}$ is defined by $\phi(z)=$ $\left(\phi_{1}(z), \ldots, \phi_{n}(z)\right)$, where $\phi_{j}(z)$ are given by formal power series in $z$ without the constant term, and $\phi(z)$ preserves $\Omega$. We have

Theorem 1.2. Let $M \subset \mathbb{C}^{2}$ be a formal surface given by (1.3). Assume that its Bishop invariant $\gamma$ satisfies $0<|\gamma|<1 / 2$ or is non-exceptional if $1 / 2<|\gamma|<$ $\infty$. Then there exists a unique formal unimodular transformation $\phi$ such that $\phi(M)$ is given by

$$
\left\{\begin{array}{l}
x_{2}=\zeta \bar{\zeta}+\left(1+\rho x_{2}^{s}\right)\left(\gamma \zeta+\bar{\gamma} \bar{\zeta}^{2}\right) \\
y_{2}=0, \quad \zeta=\alpha\left(z_{1}, x_{2}\right) z_{1}
\end{array}\right.
$$

where $\rho \in \mathbb{R} \backslash\{0\}$ and $s$ is a positive integer, or $\rho=0$ with $s=\infty$. Furthermore, $\alpha\left(z_{1}, z_{2}\right)$ satisfies the normalizing conditions:

(i) $\alpha(0)=1$ for $\rho \neq 0$; or

(ii) $\alpha\left(0, z_{2}\right)=e^{i \theta\left(z_{2}\right)}, \theta(0)=0$, and $\bar{\theta}\left(z_{2}\right)=\theta\left(z_{2}\right)$ if $\rho=0$.

The ingredients for deriving (1.4) are the Moser-Webster normal form and the group of formal automorphisms of a real analytic surface given in [5]. By considering the action of the group of automorphisms on a suitable functional space, we are able to find our normal form and to show that (1.4) is realized by a convergent unimodular transformation if and only if its Moser-Webster normal form can be realized by a convergent biholomorphic mapping. In particular, this implies that the unimodular transformation converges when $0<|\gamma|<1 / 2$. It also shows that divergence occurs when $1 / 2<|\gamma|<\infty$. One notices that $|\gamma|, s$, and the sign of $\rho$ are the full set of invariants given in [5].

\section{TOTALLY REAL SUBMANIFOLDS}

Throughout the discussion of normal forms, mappings and transformations are always defined in small open sets which are not specified. In particular, 
$f:\left(X, x_{0}\right) \rightarrow\left(Y, y_{0}\right)$ denotes a mapping which is defined near $x_{0}$ and $f\left(x_{0}\right)=$ $y_{0}$.

We now consider the normal form of a totally real and real analytic submanifold $M^{k} \subset \mathbb{C}^{n}(n \geq 2)$. For $k<n$, we want to show that $M^{k}$ is equivalent to the standard $\mathbb{R}^{k}$ by unimodular transformations. For the proof, let $\xi=\varphi(z)$ be local holomorphic coordinates such that $\varphi(0)=0$ and $\varphi(M)=\mathbb{R}^{k}$ :

$$
\operatorname{Im} \xi_{1}=\cdots=\operatorname{Im} \xi_{k}=\xi_{k+1}=\cdots=\xi_{n}=0 .
$$

One can decompose uniquely $\varphi=\sigma \circ \tau$, where $\xi=\sigma(w)$ fixes pointwise the hyperplane $w_{n}=0$, and $w=\tau(z)$ is a unimodular transformation. To see this, we put

$$
w=\sigma^{-1}(\xi)=\left(\xi^{\prime}, \xi_{n} a\left(\xi^{\prime}, \xi_{n}\right)\right), \quad \xi^{\prime}=\left(\xi_{1}, \ldots, \xi_{n-1}\right) .
$$

Denote by $D$ the Jacobian matrix for a given mapping. We have

$$
\operatorname{det}\left(D \sigma^{-1}\right)=\operatorname{det}\left(D\left(\tau \circ \varphi^{-1}\right)\right), \quad \operatorname{det}(D \tau) \equiv 1 .
$$

Hence

$$
\frac{\partial}{\partial \xi_{n}} \xi_{n} a\left(\xi^{\prime}, \xi_{n}\right)=\operatorname{det}\left(D \varphi^{-1}\left(\xi^{\prime}, \xi_{n}\right)\right)
$$

Clearly, the solution to $(2.1)$ exists uniquely. We now obtain that $\tau(M)=$ $\sigma^{-1}\left(\mathbb{R}^{k}\right)$ :

$$
\left\{\begin{array}{l}
w_{n}=r\left(x^{\prime}\right), \quad x^{\prime}=\left(\operatorname{Re} z_{1}, \ldots, \operatorname{Re} z_{k}\right) \\
\operatorname{Im} w_{1}=\cdots=\operatorname{Im} w_{k}=0, \quad w_{k+1}=\cdots=w_{n-1}=0,
\end{array}\right.
$$

where $r\left(x^{\prime}\right)$ converges near $0 \in \mathbb{R}^{k}$. Let

$$
\tau_{0}(w)=\left(w_{1}, \ldots, w_{n-1}, w_{n}-r\left(w_{1}, \ldots, w_{k}\right)\right) .
$$

Then $\psi=\tau_{0} \circ \tau$ is unimodular, and locally $\psi(M)=\mathbb{R}^{k}$.

The above argument also shows that a totally real and real analytic submanifold $M^{n} \subset \mathbb{C}^{n}$ may be locally transformed into $\mathbb{R}^{n-1} \times \mathbb{C}$ under unimodular transformations. However, there exists a functional module of obstructions to straighten $M^{n}$ to be $\mathbb{R}^{n}$.

Let $M^{n} \subset \mathbb{C}^{n}$ be a totally real and real analytic submanifold containing $p$. Since $M$ is totally real, we have $\omega_{M} \neq 0$. To describe the intrinsic property of $\omega_{M}$, we consider an arbitrary real analytic parameterization $\varphi:\left(\mathbb{R}^{n}, 0\right) \rightarrow$ $(M, p)$ and let $\omega_{\varphi}=\varphi^{*} \omega_{M}$. Obviously, $\omega_{\varphi}$ is a nowhere vanishing real analytic $n$-form. Conversely, for a nowhere vanishing $\mathbb{C}$-valued real analytic $n$ form $\omega$ defined near 0 , there exists a totally real and real analytic submanifold $\varphi:\left(\mathbb{R}^{n}, 0\right) \rightarrow\left(\mathbb{C}^{n}, 0\right)$ which realizes $\omega$. To see this, we write $\omega=a(x) d x_{1} \wedge$ $\cdots \wedge d x_{n}$. Then there exists a $\mathbb{C}$-valued real analytic function $b(x)$ given by the equation

$$
\frac{\partial b(x)}{\partial x_{n}}=a(x), \quad b(x) \equiv 0 \text { for } x_{n}=0 .
$$

We define $\varphi(x)=\left(x_{1}, \ldots, x_{n-1}, b(x)\right)$. Thus $\varphi:\left(\mathbb{R}^{n}, 0\right) \rightarrow\left(\mathbb{C}^{n}, 0\right)$ is a realization of $\omega$.

Lemma 2.1. Suppose that $M, \widetilde{M} \subset \mathbb{C}^{n}(n \geq 2)$ are two totally real and real analytic submanifolds containing 0 . Let $\varphi, \widetilde{\varphi}:\left(\mathbb{R}^{n}, 0\right) \rightarrow\left(\mathbb{C}^{n}, 0\right)$ be real 
analytic parameterizations for $M$ and $\widetilde{M}$, respectively. Then $M$ and $\widetilde{M}$ are equivalent under unimodular transformations with 0 fixed if and only if there exists a real analytic mapping $f:\left(\mathbb{R}^{n}, 0\right) \rightarrow\left(\mathbb{R}^{n}, 0\right)$ such that $f^{*} \omega_{\tilde{\varphi}}=\omega_{\varphi}$.

Proof. We first assume that there exists a unimodular transformation $F:\left(\mathbb{C}^{n}, 0\right)$ $\rightarrow\left(\mathbb{C}^{n}, 0\right)$ such that $F(M)=\widetilde{M}$. Let $f=\widetilde{\varphi}^{-1} \circ F \circ \varphi$. Then $f^{*} \omega_{\tilde{\varphi}}=$ $\omega_{\varphi}$. Conversely, given a real analytic mapping $f:\left(\mathbb{R}^{n}, 0\right) \rightarrow\left(\mathbb{R}^{n}, 0\right)$ with $f^{*} \omega_{\tilde{\varphi}}=\omega_{\varphi}$, we complexify $\tilde{\varphi} \circ f \circ \varphi^{-1}$ to be $F:\left(\mathbb{C}^{n}, 0\right) \rightarrow\left(\mathbb{C}^{n}, 0\right)$. Clearly, $\left.F^{*} \Omega\right|_{M}=\left.\Omega\right|_{M}$. Since both are holomorphic $n$-forms and $M$ is real analytic, this implies that $F^{*} \Omega=\Omega$.

To classify $\mathbb{C}$-valued real analytic $n$-forms on $\mathbb{R}^{n}$, we write $\omega=\rho \mu d x_{1} \wedge$ $\cdots \wedge d x_{n}$ where $\rho, \mu$ are real analytic, $\rho>0$, and $|\mu|=1$. By a change of real analytic coordinates, one can achieve $\rho \equiv 1$. One may also replace $\mu(0)$ by $-\mu(0)$ via reversing the orientation of $\mathbb{R}^{n}$. Therefore, we may restrict ourselves to the set of $n$-forms which have the form

$$
\omega=e^{i \theta(x)} d x_{1} \wedge \cdots \wedge d x_{n}, \quad \theta(0) \in[0, \pi),
$$

where $\theta(x)$ is real analytic. The group of transformations acting on $n$-forms (2.2) is the set of real analytic mappings which fix the origin and preserve the standard volume form on $\mathbb{R}^{n}$.

As a consequence of Lemma 2.1, we have

Corollary 2.2. Suppose that $M \subset \mathbb{C}^{n} \quad(n \geq 2)$ is a totally real and real analytic submanifold of dimension $n$ containing $p$. Let $\theta$ be defined by (1.1). Then $M$ is unimodularly equivalent to a linear subspace if and only if $\theta$ is constant near $p$. In the general position, i.e., $d \theta(p) \neq 0, M$ can be transformed into

$$
y_{1}=\cdots=y_{n-1}=\operatorname{Im} e^{-i\left(\theta(0)+x_{1}\right)} z_{n}=0
$$

through a unimodular transformation $\phi$ with $\phi(p)=0$.

We now understand that classifying totally real manifolds under unimodular transformation is the same as classifying real analytic functions under volumepreserving mappings.

Proof of Theorem 1.1. Choose a real analytic parameterization $\phi:\left(\mathbb{R}^{n}, 0\right) \rightarrow$ $\left(M^{n}, p\right)$ such that $\phi^{*} \omega_{M}$ is given by (2.2). By Vey's Morse lemma [6], there exists an analytic mapping $f:\left(\mathbb{R}^{n}, 0\right) \rightarrow\left(\mathbb{R}^{n}, 0\right)$ which preserves the standard volume form on $\mathbb{R}^{n}$ and transforms $\theta(x)$ into

$$
\tilde{\theta}(w)=\theta\left(f^{-1}(w)\right)=\sum_{k=0}^{\infty} c_{k} h(w)^{k},
$$

where $h(w)=\sum_{j=1}^{s} w_{j}^{2}-\sum_{j=s+1}^{n} w_{j}^{2}(s \geq 1)$. Furthermore, $\left\{s, c_{0}, c_{1}, \ldots\right\}$ is the full set of unimodular invariants for the function $\theta$. Hence for a volumepreserving transformation, we may assume that $(2.2)$ has the form

$$
\tilde{\omega}=e^{i \tilde{\theta}(w)} d w_{1} \wedge \cdots \wedge d w_{n} .
$$

We need to construct a mapping $\tilde{\varphi}:\left(\mathbb{R}^{n}, 0\right) \rightarrow(\tilde{M}, 0) \subset\left(\mathbb{C}^{n}, 0\right)$ such that $\tilde{\varphi}$ realizes the $n$-form (2.4), while $\tilde{M}$ has the normal form as stated in the 
theorem. To this end, let $z=\tilde{\varphi}(w)=\left(w^{\prime}, w_{n} b\left(w^{\prime}, w_{n}\right)\right)$ or

$$
w=\tilde{\varphi}^{-1}\left(z^{\prime}, z_{n}\right)=\left(z^{\prime}, z_{n} a\left(z^{\prime}, z_{n}\right)\right) \text {. }
$$

Set $\tilde{\varphi}^{*} \Omega=\tilde{\omega}$. From (2.4) and (2.5), we have

$$
\operatorname{det}\left(D \tilde{\varphi}^{-1}(z)\right)=\exp \left\{-i \tilde{\theta}\left(\tilde{\varphi}^{-1}(z)\right)\right\},
$$

which, in view of (2.3), is exactly the equation (1.2). Therefore, by (2.5), the solution to (1.2) defines a mapping $\tilde{\varphi}$ such that $\tilde{\varphi}\left(\mathbb{R}^{n}\right)$ is the normal form for the given $M$. One notes that the convergence of the solution follows easily from (2.1). Since $\left\{p, c_{0}, c_{1}, \ldots\right\}$ is the full set of unimodular invarians of $\theta$, it also provides the full set of unimodular invariants for $M$ by Lemma 2.1 . This proves the theorem.

To discuss the global invariant, we assume that $N$ is an $n$-dimensional complex manifold with a non-vanishing holomorphic $n$-form $\Omega$. Let $f: M \rightarrow N$ be a totally real immersion or embedding. Assume that $M$ is orientable and is given a volume form $\omega$. Write $f^{*} \Omega=\rho \mu \omega$, where $\rho$ and $\mu$ are smooth functions with $\rho>0,|\mu|=1$. Alternatively, $\mu$ can be defined as follows. Choose a unimodular frame $v_{1}, \ldots, v_{n}$ for $T_{x} M$. Let $e_{1}, \ldots, e_{n}$ be a unimodular frame for $T_{f(x)}^{(1,0)} N$. Then $d f_{x}\left(v_{1}, \ldots, v_{n}\right)=\left(e_{1}, \ldots, e_{n}\right) A(x)$, where $A(x) \in G(n, \mathbb{C})$. It is easy to see that $\rho(x) \mu(x)=\operatorname{det}(A(x))$. Hence $\mu(x)=\operatorname{det}(A(x)) /|\operatorname{det}(A(x))|$. Denote by $\vartheta_{f}$ the pull-back of the generator $1 \in \mathbf{H}^{1}\left(S^{1}, \mathbb{Z}\right) \simeq \mathbb{Z}$ by $\mu: M \rightarrow S^{1}$. Clearly, a regular homotopy of totally real immersions induces a homotopy for the invariant function $\mu$. This implies that, for $f: M \rightarrow N, \vartheta_{f} \in H^{1}(M, \mathbb{Z})$ is invariant under regular homotopy of totally real immersions.

We notice a standard fact due to Moser [4] that when $M$ is compact, there exists a 1-parameter family of automorphisms $\phi_{t}$ of $M$ such that

$$
\phi_{0}=\mathrm{Id}, \quad \phi_{1}^{*}(\rho \omega)=c \omega,
$$

in which $c=\int_{M} \rho \omega / \int_{M} \omega$. Therefore, for a compact and orientable manifold $M$ of volume form $\omega$, a totally real immersion $f: M \rightarrow N$ is regular homotopic to a totally real immersion $\widetilde{f}: M \rightarrow N$ which satisfies $\widetilde{f}^{*} \Omega=c \mu \omega$, where $c$ is constant. One can see that $c$ is a global unimodular invariant.

We should mention here that the above $\vartheta_{f}$ for totally real immersion $f$ : $M \rightarrow N$ has appeared in [3], where F. Forstneric defined an index homomorphism from $H_{1}(M, \mathbb{Z})$ into $\mathbb{Z}$ which is analogous to a definition of Maslov index for Lagrangian manifold given by V. I. Arnol'd [1]. Here we consider totally real immersions in an ambient complex manifold $N$ of which the canonical line bundle is trivial. In fact, when the ambient space $N$ is compact or simply connected, the cohomology class $\vartheta$ is independent of the choices of holomorphic volume forms on $N$.

\section{Normal fORMS OF MOSER AND Webster}

We will recall the Moser-Webster normal forms of real surfaces under biholomorphic transformations, which will be used in the next section.

Let $M \subset \mathbb{C}^{2}$ be a real analytic submanifold with a complex tangent at $0 \in$ $M$. For a linear unimodular change of coordinates, we may assume the $z_{1}$-axis 
is tangent to $M$ at 0 . Then $M$ is given by $z_{2}=a z_{1}^{2}+b z_{1} \bar{z}_{1}+c \bar{z}_{1}^{2}+O\left(\left|z_{1}\right|^{3}\right)$. We make a non-degeneracy assumption that $b \neq 0$. Applying the unimodular transformation

$$
\left(z_{1}, z_{2}\right) \mapsto\left(b|b|^{-\frac{2}{3}} z_{1}, b^{-1}|b|^{\frac{2}{3}} z_{2}\right),
$$

we achieve $b=1$. Hence $M: z_{2}=\tilde{a} z_{1}^{2}+z_{1} \bar{z}_{1}+\bar{\gamma} \bar{z}_{1}^{2}+O\left(\left|z_{1}\right|^{3}\right)$. By a qudratic unimodular transformation $\left(z_{1}, z_{2}\right) \mapsto\left(z_{1}, z_{2}-(\widetilde{a}-\gamma) z_{1}^{2}\right)$, we obtain

$$
M:\left\{\begin{array}{l}
z_{2}=q\left(z_{1}, \bar{z}_{1}\right)+H\left(z_{1}, \bar{z}_{1}\right), \quad H\left(z_{1}, \bar{z}_{1}\right)=O\left(\left|z_{1}\right|^{3}\right), \\
q\left(z_{1}, \bar{z}_{1}\right)=z_{1} \bar{z}_{1}+\gamma z_{1}^{2}+\bar{\gamma} \bar{z}_{1}^{2}, \quad \gamma \in \mathbb{C} .
\end{array}\right.
$$

We want to show that $\gamma$ is a unimodular invariant. To see this, consider another surface

$$
M^{\prime}: z_{2}^{\prime}=z_{1}^{\prime}{\overline{z^{\prime}}}_{1}+\gamma^{\prime} z_{1}^{\prime 2}+\overline{\gamma^{\prime}}{\overline{z^{\prime}}}_{1}^{2}+O\left(\left|z_{1}^{\prime}\right|^{3}\right)
$$

Assume that there exists a unimodular transformation $F\left(z_{1}, z_{2}\right)$ such that $F(M)=M^{\prime}$. Since $d F(0)$ preserves the $z_{1}$-axis, we have $F\left(z_{1}, z_{2}\right)=\left(a z_{1}+\right.$ $\left.b z_{2}, c z_{2}\right)+O(2)$. From the defining functions of $M$ and $M^{\prime}$, we get

$$
c\left(z_{1} \bar{z}_{1}+\gamma z_{1}^{2}+\bar{\gamma} \bar{z}_{1}^{2}\right)=|a|^{2} z_{1} \bar{z}_{1}+a^{2} \gamma^{\prime} z_{1}^{2}+\bar{a}^{2} \bar{\gamma}^{\prime} \bar{z}_{1}^{2}+O\left(\left|z_{1}\right|^{3}\right) .
$$

Since $a c=1$, the above equation implies that $c=1=a$ and $\gamma=\gamma^{\prime}$. For later use, we note that under the assumption of $\gamma=\gamma^{\prime}$, we have $\operatorname{det}(D F) \in \mathbb{R} \backslash\{0\}$ even without restricting $F$ to be unimodular. For a biholomorphic change of coordinates, $\gamma$ may be replaced by the Bishop invariant $|\gamma|$.

We now consider only cases $|\gamma| \neq 0, \frac{1}{2}$, or $1 / 2<|\gamma|<\infty$ with nonexceptional values. We need the following:

Theorem 3.1 (Moser-Webster [5]). Let $M$ be a real analytic surface given by (3.1). Then $M$ is formally equivalent to

$$
Q_{\gamma, \epsilon, s}: x_{2}=z_{1} \bar{z}_{1}+\left(1+\epsilon x_{2}^{s}\right)\left(\gamma z_{1}^{2}+\bar{\gamma} \bar{z}_{1}^{2}\right), \quad y_{2}=0,
$$

of which $s$ is a positive integer with $\epsilon= \pm 1$, or $\epsilon=0(s=\infty)$. The full set of formal invariants of $M$ near 0 is given by $\{|\gamma|, \epsilon, s\}$. Denote by $\operatorname{Aut}\left(Q_{\gamma, \epsilon, s}\right)$ the group of formal automorphisms of $Q_{\gamma, \epsilon, s}$. Then $\operatorname{Aut}\left(Q_{\gamma, 0, s}\right)$ consists of transformations in the form:

$$
\left(z_{1}, z_{2}\right) \mapsto\left(z_{1} a\left(z_{2}\right), z_{2} a^{2}\left(z_{2}\right)\right), \quad a\left(z_{2}\right)=\bar{a}\left(z_{2}\right), a(0) \neq 0 .
$$

In the case $\epsilon \neq 0, \operatorname{Aut}\left(Q_{\gamma, \epsilon, s}\right)=\left\{1, \sigma \mid \sigma\left(z_{1}, z_{2}\right)=\left(-z_{1}, z_{2}\right)\right\}$.

\section{NORMAL FORMS FOR UNIMODULAR TRANSFORMATIONS}

We first discuss the formal theory of normal forms under unimodular transformations. Therefore, surfaces and transformations are given by formal power series.

Fix a formal surface $M \subset \mathbb{C}^{2}$. Assume that $0 \in M$ is a non-degenerate complex tangent. Then by a unimodular transformation we may assume that $M$ is normalized into (3.1). As we have seen in section $3, \gamma$ in (3.1) is a unimodular invariant. Therefore, we need only seek a classification of surfaces which have the form (3.1) with a fixed $\gamma$. Denote by $\mathfrak{S}(M)$ the set of formal surfaces which are equivalent to $M$ by formal transformations. Let $\mathfrak{U}$ be the group of formal unimodular transformations which preserve the quadratic part 
of (3.1). Then $\mathfrak{U}$ acts on $\mathfrak{S}(M)$ in the obvious way. Denote by $\mathfrak{S}(M) / \sim$ the equivalence classes of the action of $\mathfrak{U}$ on $\mathfrak{S}(M)$.

In section 3 we showed that if a biholormorphic mapping $z^{\prime}=\phi(z)$ leaves $\mathfrak{S}(M)$ invariant, then $\operatorname{det}(D \phi)(0) \in \mathbb{R} \backslash\{0\}$. Let $\mathfrak{F}$ be all formal power series of complex coefficients with the constant term in $\mathbb{R} \backslash\{0\}$. Denote by $\operatorname{Aut}(M)$ the group of formal automorphisms of $M$, i.e., all the formal mappings $\psi$ : $\left(\mathbb{C}^{2}, 0\right) \rightarrow\left(\mathbb{C}^{2}, 0\right)$ such that $\psi(M)=(M)$. Consider the action of $\operatorname{Aut}(M)$ on $\mathfrak{F}$ defined by

$$
(f, \psi) \mapsto f \circ \psi \cdot \operatorname{det}(D \psi), \quad f \in \mathfrak{F}, \psi \in \operatorname{Aut}(M) .
$$

Let $\mathfrak{F} / \sim$ be the equivalence classes of action $\operatorname{Aut}(M)$ on $\mathfrak{F}$. For $M^{\prime} \in \mathfrak{S}(M)$, we choose a formal mapping $\phi$ such that $\phi(M)=M^{\prime}$. Set $l\left(\left[M^{\prime}\right]\right)=[\operatorname{det}(D \phi)]$, in which [ ] stands for the equivalence class.

Lemma 4.1. $l: \mathfrak{S}(M) / \sim \rightarrow \mathfrak{F} / \sim$ is bijective.

Proof. Let $\psi_{1}$ and $\psi_{2}$ be two formal mappings such that $\psi_{1}(M)$ and $\psi_{2}(M) \in$ $\mathfrak{S}(M)$. We first show that $l$ is well defined. Assume that there exists $\varphi \in$ $\mathfrak{U}: \psi_{1}(M) \rightarrow \psi_{2}(M)$. Then $\phi=\psi_{2}^{-1} \varphi \psi_{1} \in \operatorname{Aut}(M)$. Hence $\operatorname{det}\left(D\left(\psi_{2} \phi\right)\right)=$ $\operatorname{det}\left(D \psi_{1}\right)$, which implies that $\operatorname{det}\left(D \psi_{1}\right) \equiv \operatorname{det}\left(D \psi_{2}\right)$ in $\mathfrak{F} / \sim$. To show $l$ is injective, assume that there is $\phi \in \operatorname{Aut}(M)$ such that $\operatorname{det}\left(D \psi_{1}\right)=\operatorname{det}\left(D\left(\psi_{2} \phi\right)\right)$. Then $\varphi=\psi_{2} \phi^{-1} \psi_{1}^{-1} \in \mathfrak{U}$ maps $\psi_{1}(M)$ into $\psi_{2}(M)$. Clearly $\varphi \in \mathfrak{U}$, so $\psi_{1}(M) \equiv \psi_{2}(M)$ in $\mathfrak{S}(M) / \sim$. Conversely, for $f \in \mathfrak{F}$, we consider a mapping $\psi$ given by

$$
\psi\left(z_{1}, z_{2}\right)=\left(c \beta\left(z_{1}, z_{2}\right) z_{1}, c^{2} z_{2}\right), \quad c \in \mathbb{R} \backslash\{0\}, \beta(0)=1 .
$$

Then $\psi$ is uniquely determined by $\operatorname{det}(D \psi)=f$. Clearly, $\psi(M)$ is a realization for $f$.

We need to identify $\mathfrak{F} / \sim$ with suitable normal forms.

Lemma 4.2. Let $M=Q_{\gamma, \epsilon, s}$. Then for $f \in \mathfrak{F}$ there exists a unique $\phi \in \operatorname{Aut}(M)$ such that $\tilde{f}=f \circ \phi \cdot \operatorname{det}(D \phi)=r \alpha(z)$, while constant $r$ and formal power series $\alpha(z)$ satisfy the following normalizing conditions:

(a) $r>0$ and $\alpha(0)=1$ for $\epsilon \neq 0$; or

(b) $r=1$ and $\alpha\left(0, z_{2}\right)=e^{i \theta\left(z_{2}\right)}, \theta(0)=0$, and $\bar{\theta}\left(z_{2}\right)=\theta\left(z_{2}\right)$, if $\epsilon=0$.

Proof. We need to apply Theorem 3.1. The proof for (a) is obvious, namely, we take $\phi=$ Id for $f(0)>0$, or $\phi\left(z_{1}, z_{2}\right)=\left(-z_{1}, z_{2}\right)$ if $f(0)<0$.

For (b), write uniquely

$$
f\left(0, z_{2}\right)=r_{0}\left(z_{2}\right) e^{i \theta_{0}\left(z_{2}\right)},
$$

where both $r_{0}\left(z_{2}\right)$ and $\theta_{0}\left(z_{2}\right)$ have real coefficients and $\theta_{0}(0)=0$. Let $\phi \in$ Aut $(M)$. Then from (c) in Theorem 3.1, we have

$$
\phi\left(z_{1}, z_{2}\right)=\left(a\left(z_{2}\right) z_{1}, a^{2}\left(z_{2}\right) z_{2}\right)
$$

for some power series $a\left(z_{2}\right)$ with real coefficients and $a\left(z_{2}\right) \neq 0$. Set

$$
\begin{aligned}
\tilde{f}\left(0, z_{2}\right) & =f \circ \phi \cdot \operatorname{det}(D \phi)\left(0, z_{2}\right) \\
& =f\left(0, a^{2}\left(z_{2}\right) z_{2}\right)\left(a^{3}\left(z_{2}\right)+2 z_{2} a^{2}\left(z_{2}\right) a^{\prime}\left(z_{2}\right)\right) .
\end{aligned}
$$


From the normalizing condition $\tilde{f}\left(0, z_{2}\right)=e^{i \theta\left(z_{2}\right)}$, we obtain a functional equation

$$
r_{0}\left(a^{2}\left(z_{2}\right) z_{2}\right)\left(a^{3}\left(z_{2}\right)+2 z_{2} a^{2}\left(z_{2}\right) a^{\prime}\left(z_{2}\right)\right)=1,
$$

where $a\left(z_{2}\right), r_{0}\left(z_{2}\right)$ have real coefficients and non-zero constant terms.

We want to verify (b) by showing the existence and uniqueness of solution $a\left(z_{2}\right)$ to (4.2). To solve (4.2), rewrite

$$
a(t)=a(0)(1+A(t)) \quad \text { and } \quad r_{0}\left(a^{2}(0) t\right)=r_{0}(0)(1+b(t)) .
$$

Then (4.2) becomes

$$
\left\{\begin{aligned}
3 A(t)+2 t A^{\prime}(t)= & \frac{-b(t+t A(t))}{1+b(t+t A(t))} \\
& -\left(2 A(t)+A^{2}(t)\right)\left(A(t)+2 t A^{\prime}(t)\right), \\
A(0)=0=b(0) . &
\end{aligned}\right.
$$

Let $A(t)=\sum_{k=1}^{\infty} A_{k} t^{k}$ and $b(t)=\sum_{k=1}^{\infty} b_{k} t^{k}$. Solving equation (4.3), we obtain

$$
A_{k}=-\frac{b_{k}}{2 k+3}+P_{k}, \quad k=1,2, \ldots,
$$

where $P_{1}=0$, and $P_{k}$ is a polynomial in $A_{j}, b_{j}(j=1, \ldots, k-1)$ with rational coefficients. (4.4) gives the unique solution to (4.2).

Proof of Theorem 1.2. Assume that $M$ is given by (3.1). From (b) of Theorem 3.1, there exists a formal mappping $\psi$ such that $M=\psi\left(Q_{\gamma, \epsilon, s}\right)$. Let $f=$ $\operatorname{det}(D \psi)$. Applying Lemma 4.2, we have $f \equiv \tilde{f}$ in $\mathfrak{F} / \sim$, where $\tilde{f}=r \alpha(z)$, and $r, \alpha$ satisfy the normalizing condition stated in Lemma 4.2. Let

$$
\psi\left(z_{1}, z_{2}\right)=\left(r^{\frac{1}{3}} \beta\left(z_{1}, z_{2}\right) z_{1}, r^{\frac{2}{3}} z_{2}\right),
$$

in which $\beta$ is determined by $\operatorname{det}(D(\psi))=f$. Clearly, $\beta$ still satisfies the normalizing condition stated in Lemma 4.2. In view of Lemma 4.1, $M$ is equivalent to $\psi\left(Q_{\gamma, \epsilon, s}\right)$ by formal unimodular transformations. Rewrite

$$
\psi\left(z_{1}, z_{2}\right)=\left(r^{-\frac{1}{3}} \alpha\left(z_{1}, z_{2}\right) z_{1}, r^{-\frac{2}{3}} z_{2}\right) .
$$

It is easy to see that $\alpha$ satisfies the normalizing condition stated in Lemma 4.2. Clearly, $\psi\left(Q_{\gamma, \epsilon, s}\right)$ is given by (1.4) with $\rho=\epsilon r^{-\frac{2}{3} s} \in \mathbb{R} \backslash\{0\}$ for $\epsilon \neq 0$ or $\rho=0$ for $\epsilon=0$.

To show the uniqueness of normalizing unimodular transformations, we assume that there exist unimodular transformations $\varphi_{1}, \varphi_{2}:(M, 0) \rightarrow$ $\left(\psi\left(Q_{\gamma, \epsilon, s}\right), 0\right)$. Let $\phi=\psi^{-1} \varphi_{2} \varphi_{1}^{-1} \psi$. Obviously, $\operatorname{det}(D \phi(0))=1$. The uniquness given by Lemma 4.2 implies that $\phi=$ Id for the case $\epsilon \neq 0$. Hence $\varphi_{1}=\varphi_{2}$. Next we assume that $\epsilon=0$. We recall that $\phi \in \operatorname{Aut}\left(Q_{\gamma, \epsilon, s}\right)$ is given by

$$
\phi:\left(z_{1}, z_{2}\right) \mapsto\left(a\left(z_{2}\right) z_{1}, a^{2}\left(z_{2}\right) z_{2}\right) .
$$

Clearly, we have

$$
\left.\varphi_{2} \varphi_{1}^{-1} \psi\right|_{x_{1}=0}: z_{2} \mapsto b\left(z_{2}\right) z_{2},
$$


in which $b\left(z_{2}\right)$ is a power series of real coefficients. We now have

$$
\operatorname{det}(D \phi)\left(0, z_{2}\right)=\operatorname{det}\left(\psi^{-1} \varphi_{2} \varphi_{1}^{-1} \psi\right)\left(0, z_{2}\right)=e^{i\left(\theta\left(z_{2}\right)-\theta\left(b\left(z_{2}\right) z_{2}\right)\right)} .
$$

On the other hand, from $\phi\left(z_{1}, z_{2}\right)=\left(a\left(z_{2}\right) z_{1}, a^{2}\left(z_{2}\right) z_{2}\right)$, we get

$$
e^{i\left(\theta\left(z_{2}\right)-\theta\left(b\left(z_{2}\right) z_{2}\right)\right)}=a^{3}\left(z_{2}\right)+2 z_{2} a^{2}\left(z_{2}\right) a^{\prime}\left(z_{2}\right) .
$$

Since $\theta, a$, and $b$ have real coefficients, we have $a \equiv 1 \equiv b$. Therefore $\varphi_{1}=\varphi_{2}$. The theorem is proved.

Next, we will deal with the problem of convergence. Let $M$ be a real analytic surface as in Theorem 1.2. It is clear that if the normal form (1.4) is realized by convergent unimodular transformations, then $M$ can also be transformed into the normal form of Moser-Webster. It is known that when the complex tangent is hyperbolic, the normal form (3.2) may not be realized by biholomorphic mappings [5]. Therefore, the unimodular transformation in Theorem 1.2 diverges in general when the hyperbolic complex tangent occurs. On the other hand, when the complex tangent is elliptic and $\gamma \neq 0, M$ can indeed be transformed into (3.2) by biholomorphic mappings.

Proposition 4.3. Let $M$ be a real analytic surface as in Theorem 1.2. Then $M$ can be transformed into (3.2) by biholomorphic transformations if and only if the unique unimodular transformation which transforms it into (1.4) is convergent .

Proof. Assume $M$ can be transformed into (3.2) by a biholomorphic mapping $\psi(z)$. It suffices to show that the function $f(z)=\operatorname{det}(D \psi)(z) \in \mathfrak{F}$ can be put into normal form stated in Lemma 4.2 through a convergent transformation in $\operatorname{Aut}\left(Q_{\gamma, \epsilon, s}\right)$, which is trivial if $M$ is not biholomorphically equivalent to the quadric $Q_{\gamma} \equiv Q_{\gamma, 0, \infty}$. In the case $M$ is biholomorphically equivalent to the quadric $Q_{\gamma}$, we need to show that (4.3) has a convergent solution $A(t)$, while $b(t)$ depends on $f\left(z_{1}, z_{2}\right)$ and converges near $t=0$.

We need some notation. For two formal power series $p(t)=\sum_{k=0}^{\infty} p_{k} t^{k}$ and $q(t)=\sum_{k=0}^{\infty} q_{k} t^{k}$, let us denote

$$
\begin{gathered}
\hat{p}(t)=\sum_{k=0}^{\infty}\left|p_{k}\right| t^{k}, \quad[p]_{k}=p_{k}, \\
\sum_{k=0}^{\infty} p_{k} t^{k} \prec \sum_{k=0}^{\infty} q_{k} t^{k}, \quad \text { if }\left|p_{k}\right| \leq q_{k} \quad \text { for all } j .
\end{gathered}
$$

Comparing the coefficients in (4.3), we obtain

$$
\begin{aligned}
(2 k+3)[A]_{k}= & -\left[\frac{b(t+t A(t))}{1+b(t+t A(t))}\right]_{k} \\
& -\sum_{l=1}^{k-1}(2 l+1)[A]_{l} \cdot\left[2 A+A^{2}\right]_{k-l} .
\end{aligned}
$$

Hence

$$
\widehat{A(t)} \prec \frac{\widehat{b}(t+t \widehat{A}(t))}{1-\widehat{b}(t+t \widehat{A}(t))}+\widehat{A}\left(2 \widehat{A}(t)+\widehat{A}^{2}(t)\right)
$$


Consider the formal power series $A^{*}(t)$ defined by

$$
\left\{\begin{array}{l}
A^{*}(t)=\frac{\widehat{b}\left(t+t A^{*}(t)\right)}{1-\hat{b}\left(t+t A^{*}(t)\right)}+A^{*}\left(2 A^{*}(t)+A^{* 2}(t)\right), \\
A^{*}(0)=0 .
\end{array}\right.
$$

By the implicit function theorem, $A^{*}(t)$ actually converges near $t=0$. It is clear that $A(t) \prec A^{*}(t)$. Therefore, the solution to (4.3) converges, and the proof is complete.

In [5], it is proved that a real analytic surface given by (3.1) with $0<|\gamma|<$ $1 / 2$ can be transformed into the normal form (3.2) through biholomorphic mappings. Now Theorem 1.2 and Proposition 4.3 give

Corollary 4.4. Let $M \subset \mathbb{C}^{2}$ be a real analytic surface with an elliptic complex tangent at 0 . Assume that its Bishop invariant $|\gamma| \neq 0$. Then the unique formal unimodular transformation which transforms $M$ into (1.4) is convergent.

\section{ACKNOWLEDGMENTS}

I would like to express my gratitude to my advisor Sidney M. Webster for the guidance and encouragement in the preparation of this paper. I would also like to thank the referee for several suggestions which helped me improve the exposition of the paper.

\section{REFERENCES}

1. V. I. Arnol'd, On a characteristic class appearing in the quantization condition, Functional Anal. Appl. 1 (1967), 1-14.

2. E. Bishop, Differentiable manifolds in complex Euclidean space, Duke Math. J. 32 (1965), 1-22.

3. F. Forstnerič, Analytic disks with boundaries in a maximal real submanifold of $\mathbb{C}^{2}$, Ann. Inst. Fourier (Grenoble) 37 (1987), 1-44.

4. J. K. Moser, On the volume elements on a manifold, Trans. Amer. Math. Soc. 120 (1965), 286-294.

5. J. K. Moser and S. M. Webster, Normal forms for real surfaces in $\mathbb{C}^{2}$ near complex tangents and hyperbolic surface transformations, Acta Math. 150 (1983), 255-296.

6. J. Vey, Sur le lemme de Morse, Invent. Math. 40 (1977), 1-9.

Department of Mathematics, University of Chicago, Chicago, Illinois 60637

E-mail address: gong@zaphod.uchicago.edu 